Rev Biomed 2002; 13:33-36.

\title{
Efecto de diseño para 14 serovariedades de Leptospira en Yucatán, México.
}

\section{Comunicación Breve}

José C. Segura-Correa1, Victor M. Segura-Correa², José J. Solís-Calderón².

${ }^{1}$ Facultad de Medicina Veterinaria y Zootecnia, Universidad Autónoma de Yucatán, Mérida, Yucatán, México. ${ }^{2}$ Centro de Investigación Regional del Sureste, Mocochá, Yucatán, México.

\section{RESUMEN.}

Introducción.- El tamaño de muestra para estimar la prevalencia de una enfermedad es generalmente mayor para un muestreo por conglomerados en comparación con el muestreo simple aleatorio, debido a la correlación de individuos dentro de conglomerados. El número de veces que el tamaño de muestra aumenta depende del efecto de diseño (D). El objetivo de este trabajo fue estimar los coeficientes de correlación intraconglomerados $\left(\mathrm{r}_{\mathrm{ho}}\right)$ y D para 14 serovariedades de Leptospira en el estado de Yucatán, México.

Materiales y Métodos.- Se realizaron dos estudios sección cruzada con muestreo en dos etapas y un número constante de individuos (7 vacas) por rancho. El primer estudio se realizó en la zona ganadera de abril a junio de 1997 (53 ranchos) y el segundo en las zonas agrícola y henequenera de septiembre a diciembre de 1998 (52 ranchos). Las muestras de sangre se colectaron de la vena coccígea de cada animal y los sueros se diluyeron 1:100, utilizando la prueba de aglutinación microscópica contra 14 antígenos de serogrupos de L interrogans. Se consideraron sueros positivos aquellos que desarrollaron una aglutinación del $50 \%$ o más. Los valores de $r_{\text {ho }}$ se estimaron con las componentes de varianza obtenidas a través de procedimientos de máxima verosimilitud restringida. El valor de D se obtuvo con la fórmula $\mathrm{D}=1+(\mathrm{b}-1) \mathrm{r}_{\mathrm{ho}}$, donde $\mathrm{b}$ es el número promedio de vacas que se muestrearon por rancho.

Resultados y Discusión. Los valores de $r_{\text {ho }}$ estuvieron dentro del intervalo 0.10 a 0.52 , lo que indica que conglomerados de 7 vacas requieren tamaños de muestra 1.7 a 4.6 veces más grandes que para el caso de un muestreo simple al azar. Los mayores efectos de diseño correspondieron a L. javanica, L. wolffi, L. hardjo y L. tarassovi. Se

Solicitud de sobretiros: Dr. José Segura-Correa, Fac. de Medicina Veterinaria y Zootecnia, Universidad Autónoma de Yucatán, Apdo. Postal 5-116 Itzimná, C.P. 97100, Mérida, Yucatán, México. E-mail: segura52@hotmail.com

Recibido el 26/Enero/2001. Aceptado para publicación el 21/Mayo/2001. 


\section{JC Segura-Correa, VM Segura-Correa, JJ Solís-Calderón.}

recomienda obtener estimadas de $r_{\text {ho }}$ y D para diferentes enfermedades en el trópico y en diferentes sistemas de producción para facilitar la planeación de encuestas futuras.

(Rev Biomed 2002; 13:33-36)

Palabras clave: Leptospira, efecto de diseño, correlación intraconglomerados, trópico.

\section{SUMMARY.}

\section{Design effect for 14 leptospira serovars in Yucatan, Mexico.}

Introduction. The sample size to estimate the prevalence of a disease is generally greater for a cluster sampling design than for a simple sampling design, due to the intracluster correlation. The number of times the sample size increases depends on the design effect (D). The objective of this study was to estimate the intracluster correlation and D for 14 serovars of Leptospira in state of Yucatan, Mexico.

Material and Methods. Two cross-sectional studies with two sampling stages and a constant number of animals ( 7 cows) per cluster were carried out. The first study was done in the livestock region of Yucatan from April to June of 1997 (53 ranchs) and the second one in the agricultural and central regions from September to December of 1998 (52 ranchs). Blood samples were collected from the coccigeal vein of each animal and sera were diluted 1:100 using an microscopic aglutination test against 14 antigens of $\mathrm{L}$ interrogans serogroups. Positive sera were those with a $50 \%$ or more aglutination. The values for $r_{h o}$ were calculated from variance components using restricted maximum likelihood procedures. The design effect values were calculated with the formula $\mathrm{D}=1+(\mathrm{b}-1) \mathrm{r}_{\mathrm{ho}}$, where $\mathrm{b}$ refers to the average number of cows sampled per ranch.

Results and Discussion. The $r_{\text {ho }}$ values ranged from 0.10 a 0.52 , which indicates that clusters of 7 cows required sample sizes 1.7 to 4.6 times greater than for the simple sampling design. The greater design effects corresponded to L. javanica, L. wolffi, L. hardjo and L. tarassovi. It is recommended that estimates for $\mathrm{r}_{\mathrm{ho}}$ and $\mathrm{D}$ for different diseases in the tropics and in different production systems be obtained to facilitate the planning of future surveys.

(Rev Biomed 2002; 13:33-36)

Key words: Design effect, leptospira, intracluster correlation, tropics.

\section{INTRODUCCIÓN.}

La información de la prevalencia de una enfermedad en una población es generalmente obtenida a través de estudios sección cruzada o transversales. Sin embargo, debido a la limitada disponibilidad de marcos de muestreo y altos costos de transporte es usualmente imposible e impráctico seleccionar una muestra simple aleatoria de individuos de una población. La única solución en la mayoría de las encuestas es tomar una muestra de conglomerados (grupo de individuos), donde el hato o comunidad es la unidad de muestreo y seleccionar aleatoriamente un número de animales de cada hato ó comunidad.

Para estimar el tamaño de muestra apropiado se requiere conocer la precisión (máximo error permitido en el estimador) deseada en la encuesta. El cálculo del tamaño de muestra para estimar una prevalencia en un muestreo simple aleatorio esta dado por la siguiente fórmula (1):

$n=\frac{z^{2} p q}{d^{2}}$

donde: $p$ es la prevalencia esperada; $d$ es la precisión deseada y z es el valor crítico de la tabla de la distribución normal estándar con el nivel de confianza deseado (95 o 99\%). Sin embargo, para un número dado de individuos, la precisión de una muestra de conglomerados es menor a la obtenida a través de un muestreo aleatorio simple, debido a la correlación intraconglomerados (tasa de homogeneidad); es decir, 20 animales de un mismo

\section{Revista Biomédica}


Efecto de diseño para serovariedades de Leptospira.

hato proporcionan menos información que 20 animales de diferentes hatos. Esta reducción debería ser considerada al calcular el tamaño de muestra en un muestreo por conglomerados o etapas múltiples. Una medida del incremento del error estándar de la prevalencia debido al procedimiento de muestreo utilizado está dado por la raíz cuadrada del efecto de diseño, $\mathrm{D}=1+$ (b1) $\mathrm{r}_{\mathrm{ho}}$ donde: $\mathrm{b}$ es el número promedio de individuos medidos por conglomerado $\mathrm{y}_{\mathrm{ho}}$ es el coeficiente de correlación intraconglomerados de la enfermedad en estudio (2). En un muestreo simple de conglomerados, $r_{h o}$ es equivalente a la correlación intraclase (3).

En muestreos por conglomerados la decisión que usualmente tiene que ser considerada es, cuantos conglomerados de tamaño b serán necesarios para obtener la precisión deseada, ya que $b$ suele estar limitado por el tamaño del hato y otras consideraciones prácticas. Si el efecto de diseño es conocido, el número de conglomerados (c) está dado según Bennett y col. (2):

$$
c=\frac{\frac{D z^{2} p q}{d^{2}}}{b}=\frac{D n}{b}
$$

donde: $\mathrm{n}$ es el tamaño de muestra para un muestreo simple aleatorio.

Desafortunadamente, el efecto de diseño raramente se conoce antes de realizar el estudio transversal porque $\mathrm{r}_{\mathrm{ho}}$ en la población se desconoce. Sin embargo aun cuando la fórmula $\mathrm{D}=1+(\mathrm{b}-1) \mathrm{r}_{\mathrm{ho}}$ proporcione una estimada burda del efecto de diseño, es probable que proporcione un mejor tamaño de muestra que el valor de 2 comúnmente usado para $\mathrm{D}$, independientemente del tamaño del conglomerado o tipo de enfermedad (1). Valores aproximados de $r_{h o}$ pueden obtenerse de una muestra piloto o de resultados de encuestas previas, considerando que $\mathrm{r}_{\mathrm{ho}}$ variará de región a región y de encuesta a encuesta.

El propósito de este estudio es reportar valores de $r_{\text {ho }}$ y D para 14 serovariedades de Leptospira derivados de los resultados de una encuesta realizada en el estado de Yucatán, México.

\section{MATERIALES Y MÉTODOS.}

El estado de Yucatán se localiza al sureste de México entre las coordenadas $1930^{\prime}$ y 21 35' latitud norte y 9024 ' altitud oeste del meridiano de Greenwich. El clima es tropical subhúmedo con lluvias en verano. Los promedios de temperatura y humedad relativa son de $26.6 \mathrm{C}$ y $78 \%$, respectivamente. La precipitación pluvial varia de 415 a $1290 \mathrm{~mm}$ dependiendo del área (4). El sistema ganadero predominante es el semiintensivo, basado principalmente en el pastoreo de Guinea (Panicum maximum) y Estrella de Africa (Cynodon plectostachyus), con suplementación durante la época de seca.

Se realizaron dos estudios sección cruzada con muestreo en dos etapas y un número constante de individuos (7 vacas) por rancho. El primer estudio se realizó en la zona ganadera de abril a junio de 1997 (53 ranchos) y el segundo en las zonas agrícola y henequenera de septiembre a diciembre de 1998 (52 ranchos). Las muestras de sangre se colectaron de la vena coccígea de cada animal. Los sueros fueron diluidos 1:100 y se utilizó la prueba de aglutinación microscópica (5) contra 14 antígenos de serogrupos de L interrogans. Se consideraron sueros positivos aquellos que desarrollaron una aglutinación del $50 \%$ o más.

Los coeficientes de correlación intraconglomerados se calcularon utilizando la fórmula:

$$
r_{h o}=\frac{\sigma_{B}^{2}}{\sigma_{B}^{2}+\sigma_{W}^{2}}
$$

Donde: $\sigma_{B}^{2}$ es la componente de varianza debida a los individuos y $\sigma_{W}^{2}$ es la componente de varianza residual. Los análisis de hicieron con opción REML del procedimiento VARCOMP del paquete estadístico SAS (6). El valor de D fue obtenido utilizando la fórmula $\mathrm{D}=1+(\mathrm{b}-1) \mathrm{r}_{\mathrm{h}}$. 
JC Segura-Correa, VM Segura-Correa, JJ Solís-Calderón.

Cuadro 1

Coeficiente de correlación intraconglomerados $\left(r_{\text {ho }}\right)$ y efecto de diseño (D) para 14 serovariedades de Leptospira interrogans en vacas del estado de Yucatán, México.

\begin{tabular}{lcc}
\hline Serovariedad & $\mathrm{r}_{\mathrm{ho}}$ & $\mathrm{D}$ \\
\hline javanica & 0.5192 & 4.58 \\
wolffi & 0.3170 & 3.18 \\
hardjo & 0.2809 & 2.94 \\
tarassovi & 0.2803 & 2.93 \\
hebdomadis & 0.2689 & 2.85 \\
panama & 0.2679 & 2.84 \\
bratislava & 0.2633 & 2.81 \\
botavia & 0.2085 & 2.44 \\
canicola & 0.1961 & 2.35 \\
pomona & 0.1960 & 2.34 \\
grippothyphosa & 0.1570 & 2.08 \\
sherman & 0.1235 & 1.85 \\
icterohamorrhagiae & 0.1029 & 1.71 \\
pyrogen & 0.0985 & 1.68 \\
\hline
\end{tabular}

\section{RESULTADOS Y DISCUSIÓN.}

Los resultados se presentan en el cuadro 1. Los valores de $r_{\text {ho }}$ estuvieron dentro del intervalo 0.10 a 0.52 , lo que indica que conglomerados (ranchos) de 7 vacas requieren tamaños de muestra 1.7 a 4.6 veces más grandes que para el caso de un muestreo simple al azar. Los mayores efectos de diseño correspondieron a L. javanica, L. wolffi, L. hardjo y L. tarassovi siendo éstos diferentes a los notificados en Colombia por Orjuela y col. (7) y Otte y Gumm (8). Estos autores obtuvieron valores de D de: 1.70, 2.04, 1.96, 2.23 y 1.87 para $\mathrm{L}$ hardjo, L pomona, L grippotyphosa, L icterohaemorrhagiae y L canicola, respectivamente comparados con valores de: 2.94, 2.34, 2.08, 1.71 y 2.35 obtenidos en este estudio para las mismas enfermedades en el mismo orden. No se observó correlación alguna entre la prevalencia de la enfermedad y el coeficiente de correlación intraconglomerados o el efecto de diseño. El efecto de diseño depende de la diseminación de la infección tanto dentro de un hato como entre hatos (8). Según estos autores, el valor de la tasa de homogeneidad y en consecuencia el efecto de diseño tiende a ser relativamente bajos para el caso de enfermedades altamente contagiosas en áreas en donde la distribución de la enfermedad entre hatos es alta.

Parece deseable obtener estimadas de $r_{\text {ho }}$ y D para diferentes enfermedades en el trópico y en diferentes sistemas de producción para facilitar la planeación de encuestas futuras.

\section{REFERENCIAS.}

1.- Segura JC, Honhold N. Métodos de muestreo para producción y salud animal. Mérida: Universidad Autónoma de Yucatán; 2000. p. 139.

2.- Bennett S, Woods T, Liyanage WM, Smith DL. A simplified general method for cluster surveys of health in developing countries. World Health Statistics Quarterly 1991; 44: 98-106.

3.- Snedecor GW, Cochran WG. Métodos Estadísticos. México: Compañía Editorial Continental; 1978. p. xxx

4.- Instituto Nacional de Estadísitica, Geografía e Informática (INEGI). Yucatán. Consulta Electrónica de Tabulados. Resultados definitivos del VII Censo AgrícolaGanadero. Aguascalientes, México: INEGI; 1994.

5.- Cole JR, Ellinghausen HC, Rubin HL. Laboratory diagnosis of leptospirosis of domestic animals. Proc US Animal Health Assoc. 1979. 83: 189-199.

6.- SAS Institute Inc. SAS user's guide, Statistics. Cary: SAS Institute Inc., 1989. p. 846.

7.- Orjuela J, Navarrete M, Betancourt A, Roqueme L, Cortez E, Morrison RB. Salud y productividad en bovinos de la costa norte de Colombia. 2. Hallazgos serológicos, bacteriológicos y parasitológicos. World Animal Review 1991; 69: 7-14.

8.- Otte MJ, Gumm ID. Intra-cluster correlation coefficient of 20 infections calculated from results of cluster-sample surveys. Prevent Vet Med 1997; 31: 147-50.

\section{Revista Biomédica}

